www.jmscr.igmpublication.org

Index Copernicus Value: 79.54

ISSN (e)-2347-176x ISSN (p) 2455-0450

crossrefDOI: https://dx.doi.org/10.18535/jmscr/v7i2.29

\title{
A study on Quality of Life and Estimation of Biomarkers in COPD patients of a Tertiary Care Centre in Eastern Region
}

Authors

\section{Dr Arijit Nag MBBS ${ }^{1}$, Dr Soma Chakrabarti MBBS, DPH, MD (Community

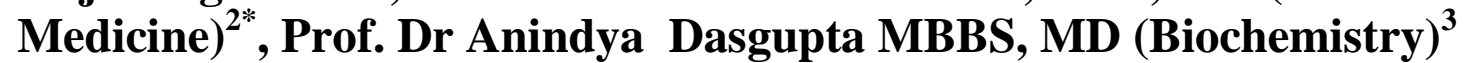

${ }^{1}$ Junior Resident, Dept of General Medicine, Calcutta National Medical College and Hospital, Kolkata

${ }^{2}$ Assistant Professor, Department of Community Medicine, Calcutta National Medical College, Kolkata

${ }^{3}$ Professor and HOD, Department of Biochemistry, Calcutta National Medical College, Kolkata

*Corresponding Author

Dr Soma Chakrabarti

E - 1/ 5, Saltee Spacio, 1, Mall Road, Kolkata - 700080

Email: c.soma20031@gmail.com, Mobile no. - +919433147313

\begin{abstract}
Chronic obstructive pulmonary disease (COPD) is a worldwide health problem. In India very few studies have been done on qualitative and quantitative assessment of COPD, despite the fact that a large and increasing portion of the population is getting affected by COPD. The aim of this study was to assess certain biomarkers in COPD patients and to determine how COPD affects Quality of life and the level of these biomarkers. The study was done on 116 COPD patients who attended a tertiary care centre in Eastern part of India. Their severity of COPD was classified as per GOLD standard. Quality of life and biomarkers levels were assessed using the SGRQ-C and appropriate biochemical methods respectively. Decreased serum nitrate level (33.67 $\pm 4.41 \mu \mathrm{mol} / \mathrm{L})$ and increased serum MDA level $(6.38 \pm 2.32 \mathrm{nmol} / \mathrm{L})$ was found, indicating increased nitrosative stress and increased oxidative stress respectively. Severity of COPD and impairment of QOL was found to be directly proportional, with significant decrease in activity component of QOL. Quality of life, as determined by the SGRQ-C, was impaired in patients with COPD. Overall it was found that quality of life deteriorated proportionately with increase in severity of COPD. There is increase in nitrosative stress which is reflected as decrease in NOS (serum nitrate) and increased oxidative stress which is expressed as high level of MDA in serum in COPD patients. Significant correlation exists between severity of COPD and serum NOS, MDA levels. Thus NOS and MDA can act as biomarkers in patients with COPD.
\end{abstract}

Keywords: COPD, NOS, MDA, QOL.

\section{Introduction}

"Chronic obstructive pulmonary disease (COPD) is a common preventable and treatable disease characterized by persistent airflow limitation that is usually progressive and associated with an enhanced chronic inflammatory response in the airways and the lung to noxious particles or gases. Exacerbations and comorbidities contribute to the overall severity in individual patient ${ }^{[1],}$. 
COPD is a major increasing global health problem with enormous amount of expenditure of direct/indirect health-care costs ${ }^{[2]}$.

COPD impairs quality of life, by preventing the affected people from leading a normal daily active life and is thus an important domain for measuring its impact. St. George's Respiratory Questionnaire (SGRQ-C), derived from the original version of SGRQ following detailed analysis of data from large studies in $\mathrm{COPD}^{[3]}$, is used to evaluate Quality of Life (QOL).

Nitrosative stress $^{[4]}$ and lipid peroxidation products $^{[5]}$ are potentially involved in COPD pathogenesis. Exhaled Nitric oxide (NO) is altered in COPD patients and serum nitrite $\left(\mathrm{NO}_{2}\right) /$ nitrate $\left(\mathrm{NO}_{3}\right)$ in patients with COPD were significantly lower than healthy controls ${ }^{[6]}$, suggesting reduced NO production or increased NO consumption (nitrosative stress) which in turn can be estimated by measuring nitrite by Griess method ${ }^{[7]}$.

Lipid peroxidation products like Malondialdehyde (MDA) can influence the inflammatory response in COPD by affecting signal transduction mechanisms, activation of redox-sensitive transcriptions factors, and chromatin regulation resulting in pro-inflammatory gene expression that lead to poor efficacy of corticosteroids in $\mathrm{COPD}^{[5]}$.

Considering the recent rise in the number of COPD patients, only a few studies have been done about serum nitrate level and MDA in COPD patients as markers of nitrosative stress and oxidative stress. Quality of life (QOL) is an important domain for measuring the impact of chronic diseases like COPD, yet there is scarcity of studies in India, particularly in West Bengal, regarding how exactly QOL is affected and how it is associated with severity of COPD. In this background the above project has been undertaken with the following objectives

a) To assess quality of life of patients in the study population.

b) To measure NOS and MDA as markers of nitrosative stress and lipid peroxidation respectively in these patients. c) To find out the association, if any, between QOL and severity of COPD.

d) To find out the association, if any, between serum biomarkers (NOS, MDA) of COPD and severity of COPD.

\section{Materials and Methodology}

Type of Study- Descriptive, observational study. Study design_- Cross-sectional study.

Study Population- All patients (both indoor and outdoor), having COPD who visited the Chest Medicine department of Calcutta National Medical College and Hospital (CNMCH).

Study Area -The study was conducted in the Departments of Chest Medicine and Biochemistry of Calcutta National Medical College and Hospital Study Duration - 1 year

Inclusion Criteria - All patients having COPD, outside the exclusion criteria, who visited the Chest Medicine department of Calcutta National Medical College and Hospital.

\section{Exclusion Criteria}

i. Patients who did not give consent.

ii. Acute exacerbation of COPD or respiratory failure.

iii. Other underlying chest diseases.

iv. Underlying heart diseases.

v. Other medical conditions that affect the QOL as diabetes mellitus, hypertension, malignancy, etc

Sample size- Sample size consisted of 116 patients (cases)

\section{Sampling technique}

a) For indoor patients no sampling technique was followed and all patients were included.

b) For outdoor patients systematic random sampling technique was followed.

\section{Study tools}

(a) St. George's Respiratory Questionnaire for COPD patients (SGRQC),

(b) Modified British Medical Research Council (mMRC) Questionnaire. 
(c) Laboratory reagents for measurement of NOS by Griess method and for MDA measurement,

(d) Pre-designed and pre-tested schedule for socio-demographic variables and other variables like biomarker level etc,

(e) Necessary instruments for spirometry, blood collection and biochemical tests.

(f) Modified B.G. Prasad scale(2013) for socio-economic status.

\section{Study technique}

(a) Interview by questionnaire,

(b) Clinical examinations - spirometry, anthropometry

(C) Laboratory investigationmeasurement of Nitric Oxide Synthase (NOS), Malondialdehyde (MDA).

(d) Records review of recent blood tests.

\section{Variables}

a) Variables for quality of life. (components determining activity score, symptoms score and impacts score)

b) Variables for lung function test Forced expiratory volume in one second $\left(\mathrm{FEV}_{1}\right)$, Forced vital capacity (FVC).

c) Variables for levels biomarkers - Nitric Oxide Synthase (NOS), Malondialdehyde (MDA).

\section{Plan of study}

After obtaining necessary ethical clearance from the Institutional Ethics Committee, the study was carried out among all patients (both indoor and outdoor), having COPD who visited the Chest Medicine department of Calcutta National Medical College and Hospital and conforming to the inclusion criteria, . The study was conducted in the Departments of Chest Medicine and Biochemistry of Calcutta National Medical College and Hospital for a period of 1 year. 116 patients were included for the study. After taking informed consent the above patients were interviewed as per the St. George's Respiratory Questionnaire for COPD patients (SGRQ-C) and the Modified British Medical Research Council (mMRC) Questionnaire. Appropriate clinical examination and review of current blood test reports was done. Blood was collected for measurement of biomarkers and sent to the Biochemistry department of $\mathrm{CNMCH}$. Forced vital capacity (FVC) and Forced expiratory volume in one second $\left(\mathrm{FEV}_{1}\right)$ readings was noted after post-bronchodilators (after applying $400 \mathrm{mcg}$ of salbutamol) spirometry. All data collected were entered into MS-Excel and analyzed using SPSS 21.

\section{Result}

The study population comprised of patients aged 45-84 years (63.1 \pm 10.01 years), of whom maximum $(55.2 \%)$ belonged to the age group 5871years. As compared to patients from rural area, urban people were found to be more affected (75.9\%). Male patients $(75.9 \%)$ were more in number and Muslim patients were the majority $(62.1 \%)$.

Most patients were just literate (29.3\%), while $10.3 \%$ patients were illiterate. It was found that both home makers and people doing unskilled labour were equally (20.7\%) affected. The study population was mostly married $(74.1 \%)$ and belonged to a nuclear family $(63.8 \%)$.

Most of the patients (48.3\%) belonged to SES Class III as per 2013 modifies BG Prasad scale. Almost $59 \%$ of the study population was addicted to tobacco or alcohol. Family history of COPD was found in $39.7 \%$ of the patients.

GOLD criteria were used to classify patients as per severity of the disease and it was found that most patients (39.7\%) belonged to "GOLD 3" or "severe" COPD. And as per mMRC, which assesses disability due to breathlessness, most of the patients $(39.7 \%)$ belonged to grade 2. [Table 1]

Upon calculating the SGRQ-C score to determine QOL, Total score was found to vary from 14.79 95.90 , with a mean score of $55.8 \pm 18.80$. Mean scores of individual components of SGRQ-C score was as follows: Symptoms score $=63.5 \pm$ 
17.45, Activity score $=60.8 \pm 14.00$, Impacts score $=50.1 \pm 22.69$. It is noteworthy that Impacts Score showed maximum range $(3.92-99.24)$, which deals with the patient's chest condition, cough and breathlessness, chest trouble and activities that may be affected by his/her breathing. [Table 2].

NOS level $(33.67 \pm 4.41 \mu \mathrm{mol} / \mathrm{L})$ was found to be decreased and MDA level $(6.38 \pm 2.32 \mathrm{nmol} / \mathrm{L})$ was found to be increased. [Table 3]

Impairment of QOL was directly proportional to the severity of COPD.
Kruskal-Wallis Test was performed to determine the association between severity of COPD and QOL. It was found that Activity Component of QOL was significantly impaired $(\mathrm{p}<0.05)$ with increasing severity of COPD. [Table 4]

Severity of COPD shows significant association with the level of biomarkers. The Spearman's correlation test shows that with increase in severity there is a significant decrease in NOS level $(\mathrm{p}=.019$, rho $=-.218)$ but significant increase in MDA level $(\mathrm{p}=.000$, rho $=$ .332).[Tables 5.1, 5.2].

Table 1: Distribution of Study Population according to different classifications of COPD ( $\mathrm{n}=116)$

\begin{tabular}{lc}
\hline GOLD classification & No.(\%) \\
\hline GOLD 1 (mild) & $30(25.9)$ \\
GOLD 2 (moderate) & $28(24.1)$ \\
GOLD 3 (severe) & $46(39.7)$ \\
GOLD 4 (very severe) & $12(10.3)$ \\
\hline mMRC classification & \\
\hline Grade 0 & No.(\%) \\
Grade 1 & $12(10.3)$ \\
Grade 2 & $22(19.0)$ \\
Grade 3 & $46(39.7)$ \\
Grade 4 & $30(25.9)$ \\
\hline
\end{tabular}

Table 2: Determination of QOL Components in the study population $(n=116)$

\begin{tabular}{lcc}
\hline QOL Components & Mean \pm Std. Deviation & RANGE \\
\hline Symptoms score & $63.5 \pm 17.45$ & $21.02-92.14$ \\
Activity score & $60.8 \pm 14.00$ & $29.48-92.45$ \\
Impacts score & $50.1 \pm 22.69$ & $3.92-99.24$ \\
Total score & $55.8 \pm 18.80$ & $14.79-95.90$ \\
\hline
\end{tabular}

Table 3: Determination of Biomarker levels in the study population $(n=116)$

\begin{tabular}{lcc}
\hline Biomarkers & Mean \pm SD & Range \\
\hline NOS $(\boldsymbol{\mu m o l} / \mathbf{L})$ & $33.67 \pm 4.41$ & $27.44-43.12$ \\
MDA $(\mathbf{n m o l} / \mathbf{L})$ & $6.38 \pm 2.32$ & $2.82-12.00$ \\
\hline
\end{tabular}

Table 4: Association between QOL and Severity of COPD in the study population $(n=116)$

\begin{tabular}{|c|c|c|c|c|c|c|}
\hline SGRQ-C Score & $\begin{array}{c}\text { Mild COPD } \\
\text { [GOLD 1] } \\
(n=15)\end{array}$ & $\begin{array}{c}\text { Moderate COPD } \\
\text { [GOLD 2] } \\
(n=14) \\
\end{array}$ & $\begin{array}{c}\text { Severe COPD } \\
\text { [GOLD 3] } \\
(\mathbf{n}=\mathbf{2 3}) \\
\end{array}$ & $\begin{array}{c}\text { Very severe COPD } \\
\text { [GOLD 4] } \\
(n=6) \\
\end{array}$ & $\begin{array}{c}\text { Kruskal } \\
\text { Wallis test }\end{array}$ & $\begin{array}{c}\mathbf{P} \\
\text { Value }\end{array}$ \\
\hline Symptoms score & $59.21 \pm 17.46$ & $68.26 \pm 16.12$ & $60.35 \pm 17.88$ & $74.93 \pm 14.70$ & 4.720 & 0.193 \\
\hline Activity score & $53.7 \pm 12.21$ & $62.30 \pm 10.24$ & $60.65 \pm 14.69$ & $75.90 \pm 12.82$ & 9.920 & 0.019 \\
\hline Impacts score & $42.64 \pm 19.07$ & $53.32 \pm 19.96$ & $48.04 \pm 24.38$ & $69.46 \pm 23.11$ & 5.011 & 0.171 \\
\hline Total score & $48.96 \pm 16.48$ & $58.72 \pm 16.02$ & $54.09 \pm 20.00$ & $72.40 \pm 18.21$ & 5.927 & 0.115 \\
\hline
\end{tabular}


Table 5.1: Association between Severity of

\begin{tabular}{|l|c|c|c|}
\hline \multirow{2}{*}{ GOLD STAGE } & \multicolumn{2}{|c|}{ NOS (in nmol/L) } & \multirow{2}{*}{$\begin{array}{c}\text { Spearman's rho } \\
\text { Correlations }\end{array}$} \\
\cline { 2 - 3 } & Mean & Range & Correlation Coefficient \\
\hline 1 & 36.21 & $29.68-41.44$ & value $=-.218^{*}$ \\
\hline 2 & 32.24 & $28.56-38.64$ & value \\
\hline 3 & 32.55 & $27.44-43.12$ & p-value $=.019$ \\
\hline 4 & 34.9 & $28.56-39.76$ & \\
\hline
\end{tabular}

COPD and NOS biomarker in the study population $(n=116)$

*Correlation is significant at the 0.05 level (2-tailed).

Table 5.2: Association between Severity of COPD and MDA biomarker in the study population ( $\mathrm{n}=116)$

\begin{tabular}{|l|c|c|c|}
\hline \multirow{2}{*}{ GOLD STAGE } & \multicolumn{2}{|c|}{ MDA (in nmol/L) } & Spearman's rho Correlations \\
\cline { 2 - 3 } & Mean & Range & \\
\hline 2 & 4.4 & $2.82-5.7$ & Correlation Coefficient \\
value $=.332^{*}$ \\
p-value $=.000$
\end{tabular}

*Correlation is significant at the 0.05 level (2-tailed).

\section{Discussion}

This study was done to assess Quality of Life and measure some biomarkers in patients with COPD in a tertiary care center in eastern region in India. The mean age of the patients, diagnosed with COPD and classified according to GOLD 2013 , was found to be $63.1 \pm 10.01$ years which is comparable to the mean age $(59.9 \pm 4.7$ years $)$ of study population of a study on the Quality of life in COPD patients in Egypt. ${ }^{[9]}$

Socio-demographic analysis of the current study showed that most patients were from urban area (75.9\%), males(75.9\%), Muslims (62.1\%), unskilled labourers1 (20.7\%), homemakers $(20.7 \%)$ and tobacco(53.5\%) or alcohol $(5.2 \%)$ addicts belonging to SES Class III(48.3\%) as per 2013 modified BG Prasad QOL as assessed with the help of SGRQ-C in the current study showed that every component of QOL was impaired. Impacts Score, which deals with the patient's chest condition, cough and breathlessness, chest trouble and activities that may be affected by his/her breathing showed maximum range (3.92 99.24). This implies that COPD hampers the normal day-to-day activity of a patient. In the study on the Quality of life in COPD patients in Egypt $^{[9]}$, similarly, QOL was assessed using the SGRQ-C and there the total score was also found to increased, hence concluding that QOL is impaired in COPD patients. In this study Impairment of QOL was found to be directly proportional to the severity of COPD. It was also found that Activity Component of QOL was significantly impaired $(\mathrm{p}<0.05)$ with increasing severity of COPD. Similar results were obtained in the study done in Egypt ${ }^{[9]}$ showed that Quality of life is impaired in patients with COPD and it deteriorates considerably with increasing severity of disease. Increasing severity of COPD is associated with a significant increase in SGRQ-C score.

In this study mean serum nitrate was measured as a marker of NOS activity and NO metabolism and was found to be $33.67 \pm 4.41 \mu \mathrm{mol} / \mathrm{L}$, which is less than the normal value. ${ }^{[12]}$. Another study done on COPD patients in Turkey [6] reported that mean levels of serum nitrite/nitrate in patients $(23.53 \pm$ $9.03 \mu \mathrm{mol} / \mathrm{L}$ ) were significantly lower than in healthy control group $(72.31 \pm 17.45 \mu \mathrm{mol} / \mathrm{L})$.So, similar trend was found in both the studies which corroborates with the role of serum nitrate as a marker of nitrosative stress in COPD.

In this study mean level of $\operatorname{MDA}(6.38 \pm$ $2.3 \mathrm{nmol} / \mathrm{L}$ ) in serum was found to be increased. MDA, a product of polyunsaturated fatty acid peroxidation is increased in COPD, which is corroborated by this study and also a similar study done in Aligarh, India ${ }^{[11]}$.

\section{Conclusion}

Quality of life, as determined by the SGRQ-C, was impaired in patients with COPD. Overall it 
was found that quality of life deteriorated proportionately with increase in severity of COPD. There is increase in nitrosative stress which is reflected as decrease in NOS (serum nitrate) and increased oxidative stress which is expressed as high level of MDA in serum in COPD patients, though no standardised scale for estimation of biomarker levels was available. Significant Correlation was noted between increase in MDA and decrease in NOS levels with increase in severity of COPD .Thus NOS and MDA can act as biomarkers in patients with COPD. Association between the biomarkers and QOL with severity of COPD can be appreciated better with future research involving a larger multicentric sample size and if the data is collected over a longer period of time.

\section{Conflicts of interest: None}

Grants availed: Self-funded

\section{References}

1. Global Initiative for Chronic Obstructive Lung Disease. Global strategy for the diagnosis, management and prevention of Chronic Obstructive Pulmonary Disease (updated 2013); 10-15.

2. Buist A, McBurnie M, Vollmer W, et al. International variation in the prevalence of COPD (the BOLD Study): a populationbased prevalence study Lancet ,2007; 370:741-50.

3. Jones P. ST GEORGE'S RESPIRATORY QUESTIONNAIRE FOR COPD PATIENTS (SGRQ-C) MANUAL, 28 September 2012; 1.2:3-4.

4. Pawar R, Abhang S, Damgaye, Lokhande R. Study Of Oxidative / Nitrosative Stress, Non-Enzymatic Antioxidants And Markers Of Airflow Obstruction (Fev1 \% Predicted) In Chronic Obstructive Pulmonary Disease (Copd) Patients. International Journal of Bioassays. March, 2014; 3 (06): 3092-3100.
5. Ilieva V, Nikolova G, Gadjeva V. Lipid Peroxidation And Catalase Activities In Patients With Chronic Obstructive Pulmonary Disease: A Comparative Study With Other Pulmonary Diseases. Trakia Journal of Sciences. 2014; 12:177-181.

6. Calikoglu M, Tamer L, Calikoglu I,Atis S, et al. Oxidative Stress and Products of Nitric Oxide Metabolism in Chronic Obstructive Pulmonary Disease and in Healthy Smokers. Turkish Respiratory Journal. 2002;3(1):2425.

7. Griess Reagent System. INSTRUCTIONS FOR USE OF PRODUCT G2930. Promega.

8. Bartoli M, Novelli F, Costa F, Bacci E, et al. Malondialdehyde in Exhaled Breath Condensate as a Marker of Oxidative Stress in Different Pulmonary Diseases. Hindawi Publishing Corporation Mediators of Inflammation. 2011;891752:1-5.

9. Zamzam M, Azab N, Wahsh R, Ragab A, Allam E. Quality of life in COPD patients. Egyptian Journal of Chest Diseases and Tuberculosis.2012;61: 281-289.

10. Brindicci C, Kharitonov S, Ito M, Elliott M, et al. Nitric Oxide Synthase Isoenzyme Expression and Activity in Peripheral Lung Tissue of Patients with Chronic Obstructive Pulmonary Disease. American Journal of Respiratory and Critical Care Medicine. 2009;181(1):21-30.

11. Waseem S, Hossain M, Rizvi S, Ahmad Z, Islam N. Oxidative Stress and Lipid Profile in COPD Patients: Beneficial Role of Exercise and Scope for Improvement. Biomedical Research.2013; 24 (1): 135-138.

12. Kawakatsu M, Ishihara $\mathrm{T}$, Kani $\mathrm{K}$, et al. Plasma Nitrate/Nitrite Concentration in Healthy Population and Patients with Diabetes Mellitus- Relationships with Gender, Aging and Diabetic Complications. Bulletin of the Osaka Medical College.2002;1(2):1-6. 